\title{
Erratum: Spectral transfer from phase to intensity in Fresnel diffraction [Phys. Rev. A 93, 053834 (2016)]
}

S. Hahn, Y. Müller, R. Hofmann, J. Moosmann, O. Öktem, L. Helfen, J.-P. Guigay, Th. van de Kamp, and T. Baumbach (Received 20 December 2016; published 9 January 2017)

DOI: 10.1103/PhysRevA.95.019901

We have noted that in Appendix D of our paper four typographical errors have occurred. The following replacements are in order: $i^{n} \rightarrow(-i)^{n}$ in Eq. (D2), $(-1)^{n} \rightarrow(-1)^{l}$ in Eq. (D3), $(-1)^{k+l} \rightarrow(-1)^{k+l+1}$ in Eq. (D8) and the last equation in (D10). 